\title{
Utilizing a Novel Pituitary Retractor for Early Descent of the Diaphragma Sellae during Endoscopic Transsphenoi- dal Pituitary Surgery
}

\author{
Jae-Sung Park, ${ }^{1}$ Dong-Sup Chung, ${ }^{2}$ Wan-Soo Yoon ${ }^{2}$ \\ Department of Neurosurgery, Seoul St. Mary's Hospital, College of Medicine, The Catholic University of Korea, Seoul, Korea \\ Department of Neurosurgery, Incheon St. Mary's Hospital, College of Medicine, The Catholic University of Korea, Seoul, Korea
}

Objective : Early descent of the diaphragm sellae (DS) during endoscopic endonasal transsphenoidal surgery (EETS) for pituitary macroadenoma surgery is occasionally a troublesome event by blocking the surgical field. Here we introduce an alternative technique with the new pituitary retractor and present our clinical experiences.

Methods : We designed a simple and rigid pituitary retractor with the least space occupation in the nasal cavity to be compatible in EETS. The pituitary retractor was held by external holder system to support the herniated DS stably. We retrospectively reviewed a clinical 22 cases of pituitary macroadenomas underwent EETS using the pituitary retractor.

Results : The pituitary retractor stably pushed up the herniated DS in all cases, and the surgeon proceeded the procedure with bimanual maneuver. The pituitary retractor was helpful to remove tumors around the medial cavernous sinus and behind the DS in 16 and seven cases, respectively. In four cases, the meticulous hemostasis was completed with the direct visualization by the DS elevation with this retractor. Gross total tumor resection was performed in 20/22 patients (91\%). The impaired visual function and hypopituitarism were improved in 18/20 (90\%) and 7/14 (50\%) patients after surgery, respectively. There was no complication related with the pituitary retractor.

Conclusion : During EETS for pituitary macroadenomas, the novel pituitary retractor reported in this study is a very useful technique when the herniated DS block the surgical field and bimanual maneuver. This pituitary retractor can help to result in the excellent surgical outcomes with minimal morbidity.

Key Words : Endoscopy · Pituitary neoplasms · Surgery · Technical report.

\section{INTRODUCTION}

Since the first introduction of endoscopic surgery for pituitary adenoma, the technique has rapidly advanced with the work of many pioneers over the last few decades ${ }^{7,13,24)}$. This has allowed endoscopic surgery to become a standard method for pituitary adenomas worldwide ${ }^{8,26)}$. Endoscopy offers better visualization of the surgical field with superior close-up and panoramic views, which results in better surgical resections and outcomes ${ }^{6,22}$. However, neurosurgeons require a learning

- Received : February 19, 2021 • Revised : April 19, 2021 • Accepted : May 14, 2021

- Address for reprints : Wan-Soo Yoon

Department of Neurosurgery, Incheon St. Mary's Hospital, College of Medicine, The Catholic University of Korea, 56 Dongsu-ro, Bupyeong-gu, Incheon 21431, Korea Tel : +82-32-280-5973, Fax : +82-32-280-5991, E-mail : yowas@catholic.ac.kr, ORCID : https://orcid.org/0000-0002-2504-4734

This is an Open Access article distributed under the terms of the Creative Commons Attribution Non-Commercial License (http://creativecommons.org/licenses/by-nc/4.0) which permits unrestricted non-commercial use, distribution, and reproduction in any medium, provided the original work is properly cited. 
period to adapt to endoscopic surgery ${ }^{18,27)}$.

During pituitary surgery, descent of the diaphragm sellae (DS) is occasionally encountered. This can block access for remnant tumor or cause cerebrospinal fluid (CSF) leak due to uneventful rupture of the DS ${ }^{9}$. To prevent early descent of the DS, resection of pituitary tumors is generally performed in a sequential manner from the inferior part to the lateral part and finishing with the superior part ${ }^{15)}$. Nonetheless, early descent of the DS can be encountered commonly, especially in large pituitary tumors ${ }^{4,12)}$. Most neurosurgeons use cottonoid patties to push up the herniated DS and visualize the surgical field. This method is useful if the DS has low pressure and a small degree of descent. However, additional hands are occasionally required with a suction cannula or ring currette to support redundant DS which surpasses cottonoid patties ${ }^{2,14}$. This makes it difficult to ensure meticulous bimanual maneuver.

To support for the herniated DS stably without the aid of additional hands, we devised a newly designed pituitary re- tractor for endoscopic endonasal transsphenoidal surgery (EETS). In this report, we first report the application of this new pituitary retractor for the complete bimanual maneuver during EETS and present clinical cases. We also discuss the advantages and limitations of this pituitary retractor.

\section{MATERIALS AND METHODS}

This study was approved by the Ethical Institutional Review Board (IRB) of Incheon St. Mary's Hospital (IRB No. OC20RAS10113) and the informed consent was waived by the regulation of IRB.

\section{Concept and design of a pituitary retractor}

The concept for the pituitary retractor was very simple : it should stably support the herniated DS with minimal disturbance in narrow nasal corridor (Fig. 1). The retractor should
(A)

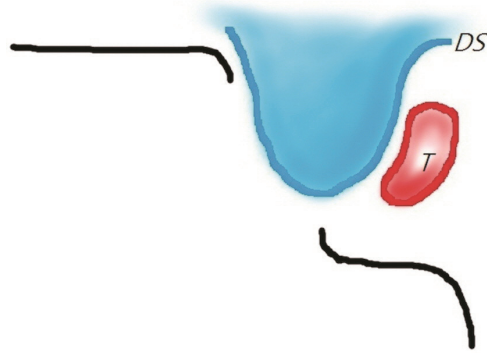

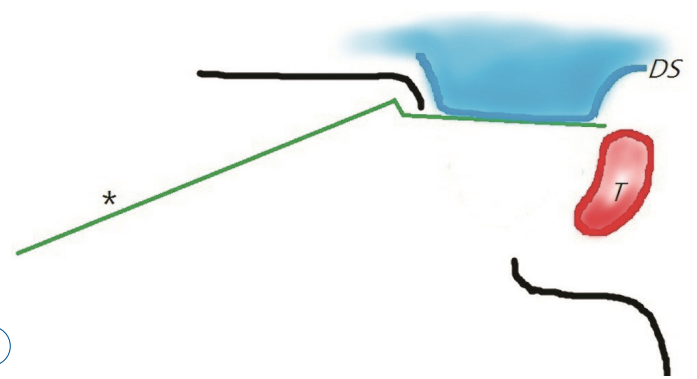

Fig. 1. Schematic illustration of pituitary retractor. A : Early descent of the diaphragma sellae (DS) into sella turcica may obscure the remaining tumor. B : With rigid support of pituitary retractor (asterisk), the DS can be elevated to reveal the concealed tumor. The remaining tumor is easily removed under direct visualization without the interruption of the ballooned DS. T : tumor.
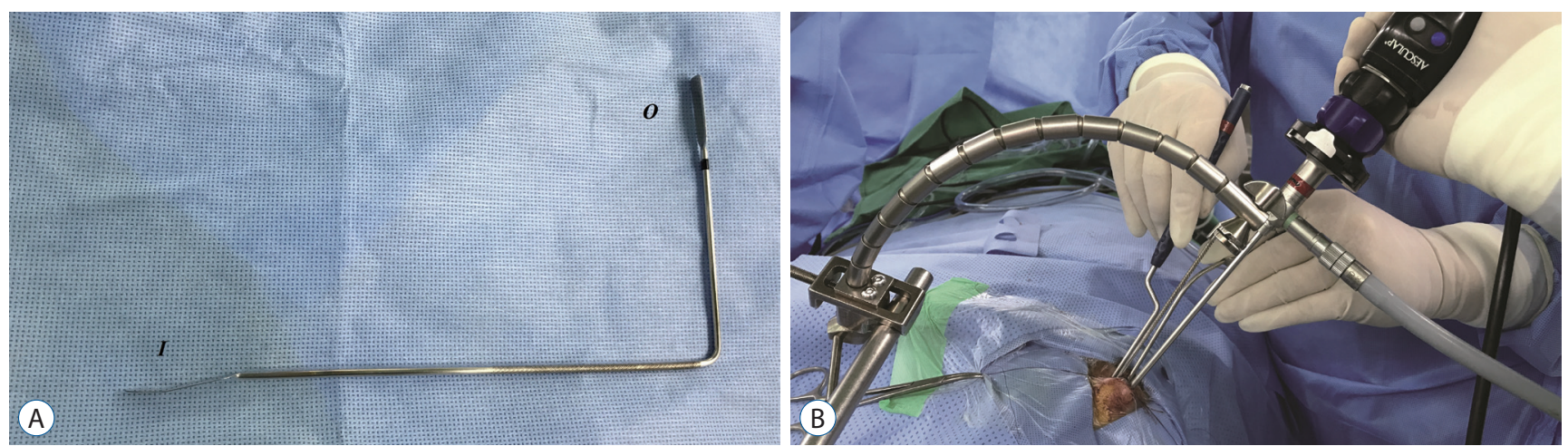

Fig. 2. Application of new pituitary retractor. A : The metallic pituitary retractor is composed of three parts. Inner part (I) has a narrow and long spatula with slightly angled head to create a working space in the sellar cavity. The middle shaft is a slender rod with a right angle to avoid interference with instruments passing through the nostril. The outer part $(0)$ is thin for fixation to the holding system. B : Pituitary retractor is introduced into left nostril and moved in the cephalic direction to create a working space below the pituitary retractor. The outer part is fixed with the Greenberg holder system (Codman, Dorchester, MA, USA). With fixation of the pituitary retractor, tumor resection is performed with bimanual maneuver. 
occupy the least space possible in the surgical route and sella cavity because instruments passaged in the narrow nostril during endoscopic endonasal approach. The retractor should also be fixed to the external device at the outside of the nose to remain unaffected by pulsatile movement of the DS. Additionally, it should be easy to operate and widely applicable. The author designed a pituitary retractor based on these ideas. A pituitary retractor is composed of three parts : inner part, shaft, and outer part. The inner part for support the descent of DS is slightly angled to create a working space below the retractor in the sella cavity. The middle shaft is slender and bent at a right angle outside the nose to avoid interference with instruments passing through the nostrils. The outer part is flattened to be held with a Greenberg holder system (Codman, Dorchester, MA, USA) (Fig. 2).

\section{Surgical procedures}

EETS for pituitary adenoma has been described in many previous literatures ${ }^{6,13)}$, and we followed the general procedures for bi-nostril EETS. A rigid endoscope (MINOP TREND, Aesculap, Tuttlingen, Germany; Image 1, Karl Storz $\mathrm{GmbH}$, Tuttlingen, Germany) was introduced through the right nostril and handled by the assistant surgeon. Principal surgeon performed the procedures to open the sphenoid sinus with suction and instruments through both nostrils. The sphenoid mucosa was denuded at sella and para-sella areas to identify bony landmarks. Wide bony removal of the sella floor was performed to include the tuberculum sella, dependent on extension of the pituitary adenoma. Tumor resection started at the inferior portion and was followed by the lateral portion. After sufficient decompression, the upper portion of the tumor attached to the DS was removed. When the DS prematurely descended to the sella cavity, tumor resection was attempted with the use of cottonoid patties to push up the DS. Despite this effort, a pituitary retractor was introduced through the left nostril when the descent of DS blocked the surgical field with the insufficient elevation with cottonoid patties alone. After the pituitary retractor was placed under the DS in the sella cavity and then pushed up toward the cephalic direction to create a working space in the sella cavity, it was held and fixed to a Greenberg holder system. With the properly placed pituitary retractor, the procedure could be performed in the bimanual procedure with increased maneuverability. This allowed wider working space without the in- terruption of the pulsatile DS movement. The operative field was re-explored to identify the remaining tumor, especially around both medical cavernous sinuses and behind the DS, and the remnant tumor was removed under direct visualization in bimanual maneuver by principal surgeon. After tumor removal, the sellar floor was reconstructed with a fibrin sealant patch, Tachosil (Baxter Healthcare Co., Deerfield, IL, USA). Abdominal facia and nasoseptal flap were used in cases with intraoperative CSF leakage. Continuous lumbar drainage for CSF diversion was also performed in selective cases with high-flow CSF leakage.

\section{Patient population}

The pituitary retractor was used in 22 patients who underwent EETS for pituitary adenomas. Patient medical records were retrospectively reviewed, including clinical data, endocrine evaluation, visual assessment, and magnetic resonance (MR) images. The surgical notes and recorded operation videos were also reviewed. Descent of the DS was investigated and classified based on a grade system as suggested by Abdelmaksoud et al. ${ }^{1)}$ : I, intrasellar view is $100 \%$ clear without descent of the DS; II, intrasellar view is $100 \%$ clear but with remarkable descent of the DS; III, intrasellar view is not clear unless the DS is pushed up by surgical instruments and cotton patties; IV, intrasellar view is not clear even with attempts to push up the descended DS; and V, herniating DS is out of the bone. Ophthalmologic examinations of best-corrected visual acuity (VA) and Humphrey visual fields (VF) were analyzed according to guidelines of the German Ophthalmological Society $^{10)}$.

The median age of included patients was 56 years (range, 31-72), and the male to female ratio was 2.1. Clinical diagnoses were nonfunctional pituitary adenomas in 19 patients, pituitary apoplexy in two, and prolactinoma in one. The preoperative median diameters were $25.5 \mathrm{~mm}$ (range, 16-34) in width and $27.5 \mathrm{~mm}$ (range, 11-42) in height. According to modified Knosp's classification, seven patients had grade I, seven patients had grade II, and eight patients had grade IIIa. Fourteen patients had preoperative hypopituitarism, while seven patients showed normal pituitary hormone status and one patient had hyperprolactinemia. Twenty patients had visual impairment with median VA score of 10 (range, 0-57) and VF score of 20 (range, 0-48) (Table 1). 


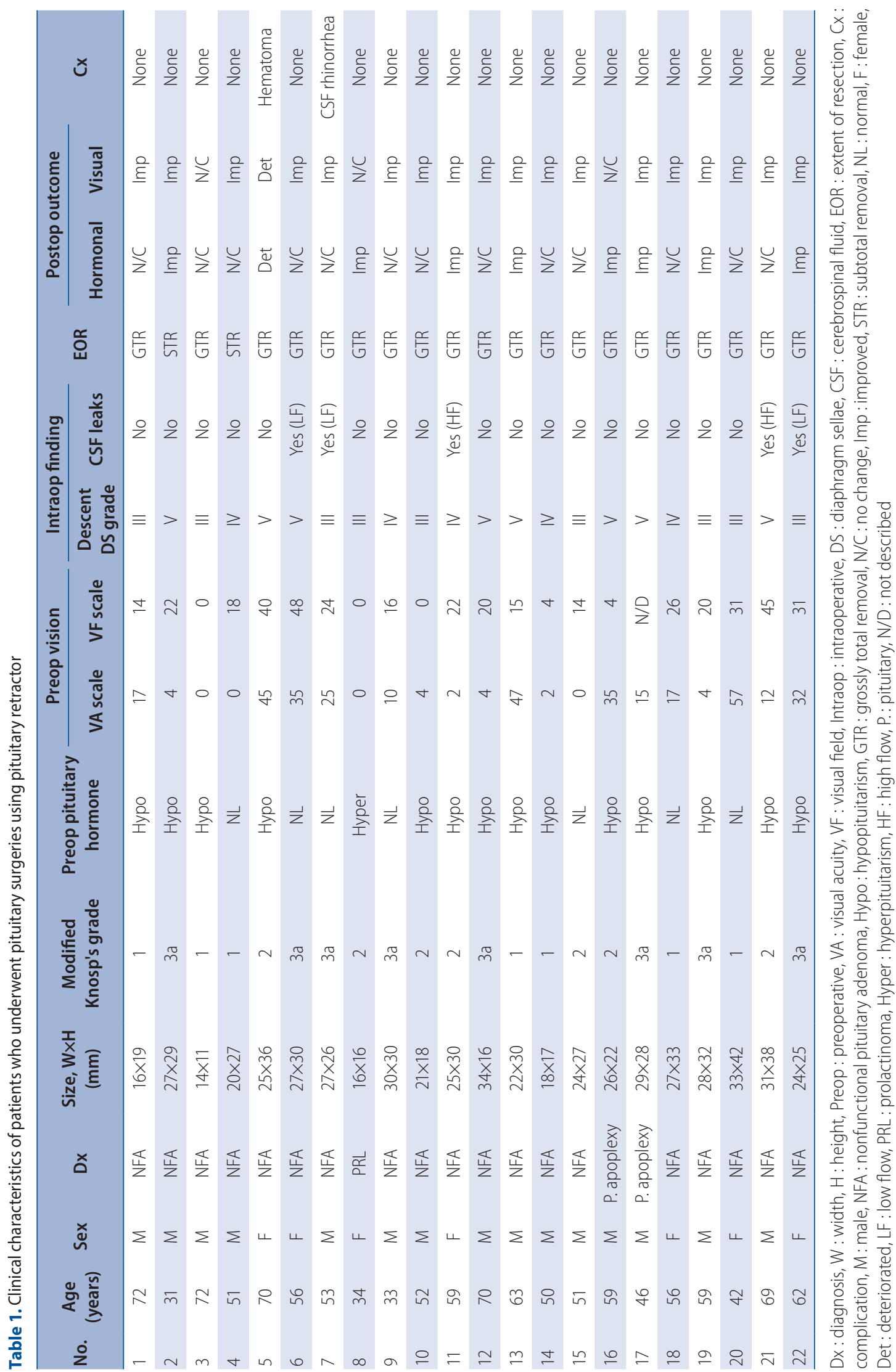




\section{RESULTS}

\section{Usefulness of pituitary retractor and extent of resection}

DS descent was considered grade III in nine patients, grade IV in five patients, and grade $\mathrm{V}$ in eight patients during tumor resection. With the help of the pituitary retractor, inspection and tumor removal around the medial cavernous sinus was performed in 16 cases. This procedure was performed by bimanual maneuver with an aspiration cannula on the surgeon's left hand and ring curette on the right hand without the interruption of the herniated DS. The retractor also acted as counter-traction to the DS descent, and remnant tumor or capsule behind redundant DS was also removed in seven cases. In four cases, the pituitary retractor was used to investigate the bleeding site with direct visualization and the meticulous hemostasis was completed with a bipolar coagulator or hemostatic materials.

Among a total of 22 patients, grossly total tumor resection was completed in 20 patients and subtotal resection was performed in two patients who underwent the operation in the early period of pituitary retractor usage. One patient with grade IIIa by modified Knosp's classification had tumor remaining around the right cavernous sinus, possibly due to scant opening of the dura (case No. 2). The other patient showed the tumor remained in the antero-superior portion on postoperative MR images and it was presumed that pituitary retractor concealed the remnant tumor because it should be positioned at the same area (case No. 4).

\section{Clinical outcome of patients}

Intraoperative CSF leakage occurred in five patients, with high-flow leaks in two and low-flow leaks in three. All patients with high-flow CSF leak underwent reconstruction of the sella floor with abdominal fascia and nasoseptal flap and had continuous lumbar drainage of $10 \mathrm{~mL}$ per hour for 7 days postoperatively. One patient was required urgent reoperation due to postoperative hematoma at the surgical field (case No. 5). One patient was re-explored due to delayed CSF rhinorrhea 14 days after operation despite the low-flow CSF leakage in the first operation (case No. 7). During the median follow-up period of 6 months (range, 1-29), postoperative visual function improved in 18 patients. One patient who underwent reoperation for postoperative hematoma had deteriorated VA.
Two other patients with normal visual function preoperatively and one other patient with blindness due to pituitary apoplexy showed no change in vision. Postoperative hormonal function of the pituitary gland was assessed for a median of 6 months (range, 1-38). Among 14 patients with preoperative hypopituitarism, three patients were within normal limits and four patients showed partial improvement of hormonal status postoperatively. One prolactinoma patient with $284.9 \mathrm{ng} / \mathrm{mL}$ of serum prolactin showed a normal prolactin level of $0.99 \mathrm{ng} / \mathrm{mL}$ at 2 days postoperative. There was no change of hormonal status in 13 patients, including seven with normal function and six with hypopituitarism. One patient with hypogonadism showed postoperative panhypopituitarism; this was the same patient with deteriorated VA.

\section{Illustrative case}

\section{Case No. 17}

A 46-year-old male patient visited the emergency room of our hospital due to headache, decreased VA and incomplete third cranial nerve palsy with preserved pupil size. The patient only had light perception in the right eye, while he had 0.6 corrected VA in the left. On initial MR images, a $21 \times 29 \times 28$ mm-sized pituitary tumor with the invasion to right cavernous sinus was shown. The patient underwent EETS, and the tumor was removed starting with internal decompression from the inferior portion. During the sequential tumor resection, the early descent of the DS was evented, and the cotton patties were attempted to support it, but the surgical field was blocked. Then, the pituitary retractor was introduced as described above to make the clear surgical field without the interruption of herniated DS and to ensure the bimanual maneuver. With the pituitary retractor, the remained tumor around the medial cavernous sinus was removed with suction cannula and ring curette under direct visualization. By using the counter-traction function of the pituitary retractor, the tumor capsule behind the DS was also removed with sharp dissection. Postoperative MR images demonstrated no remaining tumor (Fig. 3 and Supplementary Video 1). After the operation, the neurologic symptoms improved dramatically, and the patient showed 0.5/1.0 corrected VA and normal function of the third cranial nerve. 

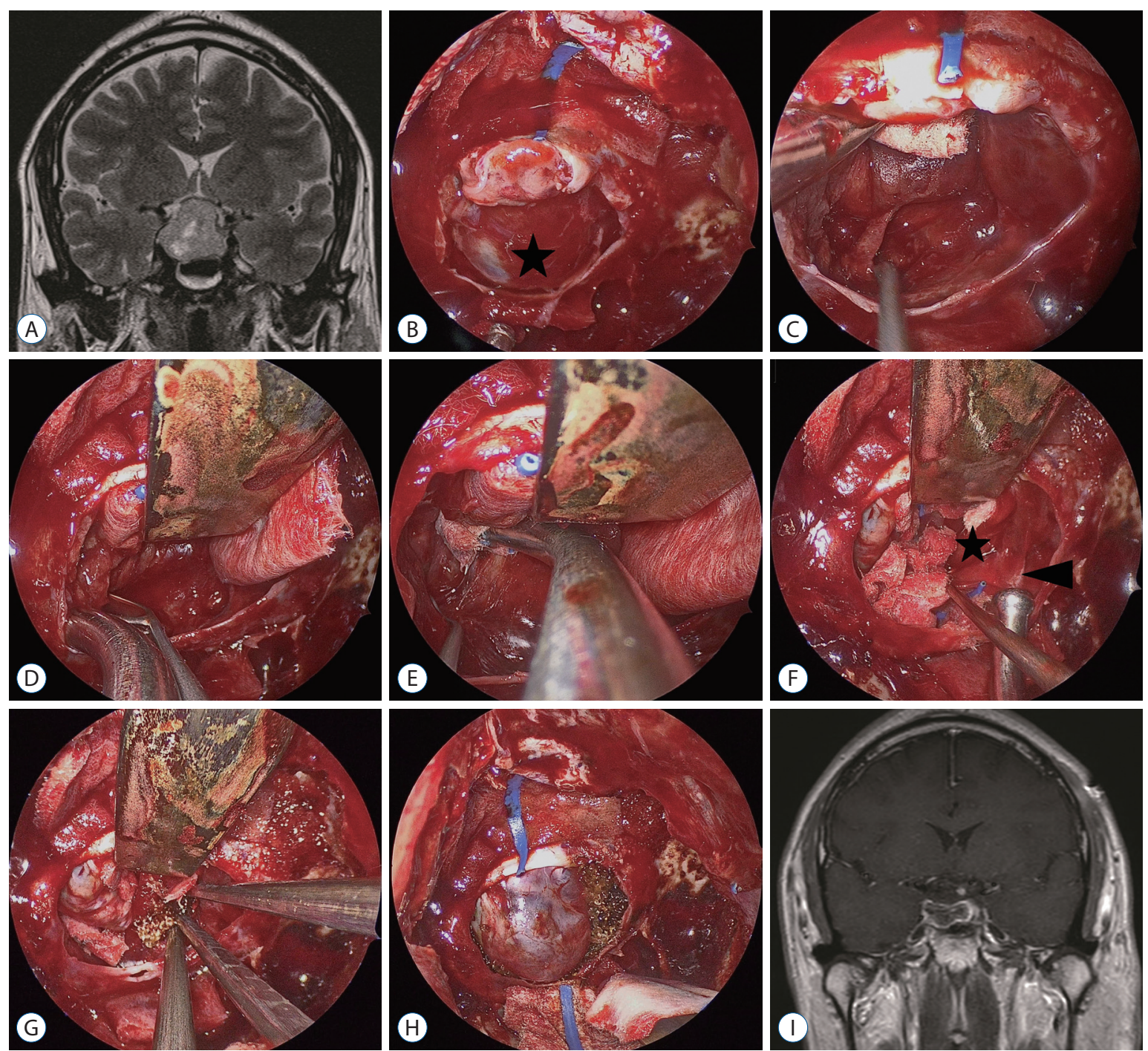

Fig. 3. Case No. 17. A : Pituitary macroadenoma compresses optic nerve superiorly and extends laterally to the right superior cavernous compartment over the intracavernous ICA. B : The diaphragma sellae (DS, black asterisk) balloons out into the sella cavity during tumor removal. $C$ : Tumor removal is proceeded using cottonoid patties to support the DS. However, the remaining tumor is not clearly identified due to the pooled blood and the limited maneuverability. $D$ and $E: A$ rigid pituitary retractor supports the DS stably, and the tumor around the right cavernous sinus is removed with a bimanual maneuver. F : Sharp dissection between the DS (black asterisk) and tumor capsule (black arrowhead) is performed under the DS. G : Hemostasis with surgicel is performed with bimanual maneuver under direct visualization. $\mathrm{H}$ : After the completion of tumor removal, the DS occupies the sella cavity where the rigid pituitary retractor is removed. I: On postoperative magnetic resonance image, the remaining tumor is not visible and the compressed optic chiasm is relieved.

\section{DISCUSSION}

The DS anatomically consists of two thin layers of dura mater, the meningeal and periosteal layers. The meningeal layer extends to cover the lateral wall and roof of the cavernous sinus superficially and the periosteal layer continues to the deep layer covering the lateral wall of the cavernous sinus ${ }^{3,28}$. During pituitary adenoma resection, the DS tends to descend to- ward the sella turcica in different patterns. Guinto Balanzar et al. ${ }^{11)}$ reported that the type of DS descent was related to tumor volume on microscopic findings. Asymmetric descent of the DS into the sella turcica was shown in large pituitary adenomas and was also related to the greatest risk of residual tumor in the surgical bed. However, the volume and height of the tumor above the diaphragm opening, not tumor volume and height, were reported to be more important determining fac- 
tors of DS descent on endoscopic findings. With larger pituitary adenomas, the DS may stretch above the diaphragm opening. This stretched DS is more liable to be redundant and prolapse during tumor resection. Additionally, residual tumor is mainly dependent on invasion to the cavernous sinus, not degree of DS descent ${ }^{1}$.

Descent of the DS into the operative field is a challenge to surgeons during EETS for pituitary adenoma. It is unclear what really descends in the surgical bed at the end of surgical resection for a pituitary adenoma because the descended structure consists of one or more of the following structures : arachnoid membrane, DS, pituitary capsule, adenoma pseudocapsule, and/or flattended pituitary gland. However, we have referred to this surgical landmark as DS in this study. This redundant DS can increase the difficulty of the operative procedure by impeding proper exploration of the surgical field. It also limits the amount of comfortable working space and bimanual maneuvers ${ }^{2,5,12,14)}$. CSF diversion with intraoperative lumbar drainage was evaluated regarding effectiveness in extent of resection by preventing bulging of the DS. Although intraoperative lumbar drainage was effective in preventing CSF leaks, the effect on extent of resection was debated and the herniated DS might increase the risk of CSF leak ${ }^{16,20,23)}$.

Neurosurgeons usually push up DS descent with cottonoid patties $^{2,14)}$. However, this can limit the bimanual maneuverability of instruments. A blind procedure is still possible depending on the degree of redundant DS. Kobayashi et al. ${ }^{17)}$ reported the microscopic transsphenoidal surgery performed with a self-retraining retractor, which was similar concept of our pituitary retractor. The bulging DS was retracted by the specially designed spatula and the surgeon could continue the procedure with both hands. However, their system can be applicable to only microscopic transsphenoidal approach because the speculum and the large corridor are mandatory. Prevedello et al. ${ }^{25)}$ used the "Q-tip" cotton swab to elevate the herniated DS gently during the endoscopic endonasal procedures. Its fine and long rod allowed it to be inserted through one nostril and make the appropriate space for the free movement of instruments during endoscopic surgery. However, a well-trained assistant surgeon is necessary because it should be held by one of surgeons. Kutlay et al. ${ }^{19)}$ published the alternative technique for early DS descent. The authors used transparent strips of polypropylene as a self-retraining retractor.
The strip was cut and tailored to fit the sella opening, and free tips of the strip were outside of the sphenoid sinus. Although successful results were reported, only three cases were reported. However, it is questionable how to remove the tumor capsule attached to the behind of descent DS. The free tips of strip outside of the sphenoid sinus is appeared to be able to interfere the movement of instruments in the narrow nostril. Additionally, it is concerned about the safety of the neurovascular structures due to the possibility of over-compression by strips itself.

We designed a new pituitary retractor for EETS that could be applied regardless of the degree of DS descent. This retractor stably supported the herniated DS with rigid metallic material held by the external holder system in all cases. It created a comfortable working space, allowing the surgical procedure to proceed without interruption by pulsation of the DS. This was helpful for removal of the remaining tumor by bimanual maneuver when the surgical field with pooled blood and/or CSF, especially around the medial cavernous sinus. Because the rigid pituitary retractor positioned at the superior area prevented downward movement of the DS, the remaining tumor behind the DS could be removed with sharp dissection by counter-traction. The bleeding point was highlighted with the aspiration cannula, and more meticulous hemostasis was performed under direct visualization. However, the retractor had some limitations. If the tumor was remained at the antero-superior portion of the DS, it could be masked by the elevated pituitary retractor due to be positioned at the same area. After this early experience, the antero-superior portion of the tumor was removed prior to application of the pituitary retractor. However, this procedure could increase the risk of CSF leak due to dehiscence between the DS and dura mater, even if low-flow CSF leakage occurred. The pituitary retractor also required a wide opening of the sella floor to prevent difficulty maneuvering instruments below the retractor. Despite these limitations, the pituitary retractor had more advantages because it allowed neurosurgeons to be free from DS descent and was easily applicable in EETS for large pituitary adenomas.

According to the systemic review about the surgical outcome for pituitary macroadenomas, a residual tumor rate of $10 \%$ to $36 \%$. Visual function and hypopituitarism were improved in $75 \%$ to $91 \%$ and in $30 \%$ to $50 \%$ of surgically treated patients, respectively. A total complication rate was $7.1 \%$, with 
CSF leak (4.7\%), meningitis (2.0\%), and vision deterioration $(2.0 \%)^{21)}$. In our study, grossly total resection was completed in 20/22 patients (91\%) with pituitary macroadenomas. 18/20 patients $(90 \%)$ with impaired visual function and $7 / 14$ patients $(50 \%)$ with hypopituitarism were improved during the postoperative period. Surgically related complication was evented in $2 / 22$ patients (9.1\%), but it was not related with pituitary retractor.

\section{CONCLUSION}

Early DS descent is a challenge during EETS for pituitary macroadenoma. Although cottonoid patties are occasionally helpful, they do not allow bimanual maneuver, if the DS has high falling pressure or if redundant DS surpasses the cottonoid patties. A new pituitary retractor reported in this study stably supports herniated DS and allows tumor removal with minimal risk by ensuring meticulous bimanual maneuver. This breakthrough allows neurosurgeons to be free from DS descent. It enhances the advantages of endoscopic surgery, such as excellent illumination, high magnification, and a panoramic view of the surgical area. This new pituitary retractor can also be helpful in the successful surgical results and the low morbidity, especially for the less experienced neurosurgeons.

\section{CONFLICTS OF INTEREST}

No potential conflict of interest relevant to this article was reported.

\section{INFORMED CONSENT}

This type of study does not require informed consent.

\section{AUTHOR CONTRIBUTIONS}

\author{
Conceptualization : WSY \\ Data curation : DSC \\ Formal analysis : JSP, WSY
}

\author{
Methodology : WSY \\ Project administration : WSY \\ Visualization : WSY \\ Writing - original draft : JSP \\ Writing - review \& editing : DSC, WSY
}

\section{ORCID}

$$
\begin{array}{ll}
\text { Jae-Sung Park } & \text { https://orcid.org/0000-0002-9827-9913 } \\
\text { Dong-Sup Chung } & \text { https://orcid.org/0000-0003-0690-4388 } \\
\text { Wan-Soo Yoon } & \text { https://orcid.org/0000-0002-2504-4734 }
\end{array}
$$

\section{- Supplementary materials}

The online-only data supplement is available with this article at https://doi.org/10.3340/jkns.2021.0047.

\section{References}

1. Abdelmaksoud A, Fu P, Alwalid O, Elazab A, Zalloom A, Xiang W, et al. : Degrees of diaphragma sellae descent during transsphenoidal pituitary adenoma resection: predictive factors and effect on outcome. Curr Med Sci 38 : 888-893, 2018

2. Bedi $A D$, Toms $S A$, Dehdashti AR : Use of hemostatic matrix for hemostasis of the cavernous sinus during endoscopic endonasal pituitary and suprasellar tumor surgery. Skull Base 21 : 189-192, 2011

3. Campero A, Martins C, Yasuda A, Rhoton AL Jr : Microsurgical anatomy of the diaphragma Sellae and its role in directing the pattern of growth of pituitary adenomas. Neurosurgery 62 : 717-723; discussion 717723, 2008

4. Cappabianca P, Alfieri A, Thermes S, Buonamassa S, de Divitiis E : Instruments for endoscopic endonasal transsphenoidal surgery. Neurosurgery 45 : 392-395; discussion 395-396, 1999

5. Cappabianca P, Cavallo LM, de Divitiis E : Endoscopic endonasal transsphenoidal surgery. Neurosurgery 55 : 933-940; discussion 940-941, 2004

6. Cappabianca P, Cavallo LM, Solari D, Stagno V, Esposito F, de Angelis M : Endoscopic endonasal surgery for pituitary adenomas. World Neurosurg 82(6 Suppl) : S3-S11, 2014

7. Cavallo LM, Somma T, Solari D, lannuzzo G, Frio F, Baiano C, et al. : Endoscopic endonasal transsphenoidal surgery: history and evolution. World Neurosurg 127 : 686-694, 2019

8. de Divitiis E, Laws ER, Giani U, Iuliano SL, de Divitiis O, Apuzzo ML : The current status of endoscopy in transsphenoidal surgery: an international survey. World Neurosurg 83 : 447-454, 2015

9. Ding ZQ, Zhang SF, Wang QH : Neuroendoscopic and microscopic trans- 
sphenoidal approach for resection of nonfunctional pituitary adenomas. World J Clin Cases 7 : 1591-1598, 2019

10. Fahlbusch $R$, Schott $W$ : Pterional surgery of meningiomas of the tuberculum sellae and planum sphenoidale: surgical results with special consideration of ophthalmological and endocrinological outcomes. J Neurosurg 96 : 235-243, 2002

11. Guinto Balanzar G, Abdo M, Mercado M, Guinto P, Nishimura E, Arechiga N : Diaphragma sellae: a surgical reference for transsphenoidal resection of pituitary macroadenomas. World Neurosurg 75 : 286293, 2011

12. Jane JA Jr, Han J, Prevedello DM, Jagannathan J, Dumont AS, Laws ER $\mathrm{Jr}$ : Perspectives on endoscopic transsphenoidal surgery. Neurosurg Focus 19 : E2, 2005

13. Jankowski R, Auque J, Simon C, Marchal JC, Hepner H, Wayoff M : Endoscopic pituitary tumor surgery. Laryngoscope 102 : 198-202, 1992

14. Jarrahy R, Berci G, Shahinian HK : Assessment of the efficacy of endoscopy in pituitary adenoma resection. Arch Otolaryngol Head Neck Surg 126 : 1487-1490, 2000

15. Jho David $H$, Jho Diana H, Jho HD : Endoscopic Endonasal Pituitary and Skull Base Surgery in Alfredo QH, Schmidek HH (eds) : Schmidek \& Sweet operative neurosurgical technique : indications, methods, and results, ed 6. Philadelphia : Saunders, 2012, Vol 1, pp257279

16. Jonathan GE, Sarkar S, Singh G, Mani S, Thomas R, Chacko AG : A randomized controlled trial to determine the role of intraoperative lumbar cerebrospinal fluid drainage in patients undergoing endoscopic transsphenoidal surgery for pituitary adenomas. Neurol India 66 : 133-138, 2018

17. Kobayashi S, Sugita K, Takemae T, Tanizaki $Y$ : Retraction system for transsphenoidal surgery. Technical note. J Neurosurg 62 : 307-309, 1985

18. Koc K, Anik I, Ozdamar D, Cabuk B, Keskin G, Ceylan S : The learning curve in endoscopic pituitary surgery and our experience. Neurosurg Rev 29 : 298-305; discussion 305, 2006

19. Kutlay $M$, Gönül E, Düz B, Izci $Y$, Tehli $O$, Temiz $C$, et al. : The use of a simple self-retaining retractor in the endoscopic endonasal transsphenoidal approach to the pituitary macroadenomas: technical note. Neurosurgery 73 Suppl Operative 2 : ons206-ons209; discussion ons209-ons210, 2013

20. Liu B, Wang Y, Zheng T, Liu S, Lv W, Lu D, et al. : Effect of intraoperative lumbar drainage on gross total resection and cerebrospinal fluid leak rates in endoscopic transsphenoidal surgery of pituitary macroadenomas. World Neurosurg 135 : e629-e639, 2020

21. Lucas JW, Bodach ME, Tumialan LM, Oyesiku NM, Patil CG, Litvack Z, et al. : Congress of neurological surgeons systematic review and evidencebased guideline on primary management of patients with nonfunctioning pituitary adenomas. Neurosurgery 79 : E533-535, 2016

22. Mamelak AN : Pro: endoscopic endonasal transsphenoidal pituitary surgery is superior to microscope-based transsphenoidal surgery. Endocrine $47:$ 409-414, 2014

23. Mehta GU, Oldfield EH : Prevention of intraoperative cerebrospinal fluid leaks by lumbar cerebrospinal fluid drainage during surgery for pituitary macroadenomas. J Neurosurg 116 : 1299-1303, 2012

24. Park JH, Choi JH, Kim YI, Kim SW, Hong YK : Modified graded repair of cerebrospinal fluid leaks in endoscopic endonasal transsphenoidal surgery. J Korean Neurosurg Soc 58 : 36-42, 2015

25. Prevedello DM, Kassam AB, Gardner P, Zanation A, Snyderman CH, Carrau RL : "Q-tip" retractor in endoscopic cranial base surgery. Neurosurgery 66 : 363-366; discussion 366-367, 2010

26. Rolston JD, Han SJ, Aghi MK : Nationwide shift from microscopic to endoscopic transsphenoidal pituitary surgery. Pituitary $19: 248-250$, 2016

27. Shikary T, Andaluz N, Meinzen-Derr J, Edwards C, Theodosopoulos P, Zimmer LA : Operative learning curve after transition to endoscopic transsphenoidal pituitary surgery. World Neurosurg 102 : 608-612, 2017

28. Songtao Q, Yuntao L, Jun P, Chuanping H, Xiaofeng S: Membranous layers of the pituitary gland: histological anatomic study and related clinical issues. Neurosurgery 64 Suppl 3 : ons1-ons9; discussion ons9-ons10, 2009 